\title{
STUDY OF THE OLIGOMERIZATION OF 1-OCTENE CATALYZED BY MACRORETICULAR ION-EXCHANGE RESINS
}

\author{
R. Bringué*, M. Cadenas, C. Fité, M. Iborra, F. Cunill \\ Chemical Engineering Department, Faculty of Chemistry, University of Barcelona, \\ C/ Martí i Franquès 1-11 08028, Barcelona, Spain
}

*Corresponding author: tel.: +34 934020155, fax: +34 934021291, e-mail address:

\section{rogerbringue@ub.edu}

\begin{abstract}
Oligomerization of 1-octene is a feasible alternative to improve the properties and the quality of fuels and lubricants oils. The performance of macroreticular ion-exchange resins as catalysts for such reaction has been evaluated. The experimental setup consisted of a batch stirred-tank reactor that operated at 353-393 K and $2 \mathrm{MPa}$. 1-octene conversion was practically complete and the selectivity to double-bond isomerization and dimers after 6 hours at $373 \mathrm{~K}$ was up to $95 \%$, and $12 \%$, respectively. The accessible acidity of the catalysts was the most important structural parameter for these reactions. Selectivity to dimers and branched isomers increased with increasing temperature. Cracking compounds were not detected at all. The theoretical equations derived from the proposed kinetic model fit well the experimental results.

Keywords: 1-octene oligomerization, ion-exchange resins, kinetics
\end{abstract}

\section{Introduction}

Gasoline composition is in a continuous evolution due to technical and ecological issues. Major efforts in oil industry are dedicated to improve fuel properties and quality and, at the same time, to fulfill environmental regulations. Oligomerization of linear 
olefins is an alternative for obtaining heavy olefins with great flexibility in its composition. The obtained products can be added to gasoline, diesel and lubricating oils.

The available literature on oligomerization of olefins is in reverse order to the number of carbon atoms. A great deal of references can be found for ethylene and propylene, many for butylenes and very scarce for pentenes and heavier ones. The number of existing commercial plants follows the same tendency. Oligomerization of light olefins $\left(\mathrm{C}_{3}-\mathrm{C}_{5}\right)[1-4]$ is widely found in the literature. Some works have recently appeared concerning $\mathrm{C}_{6}$ olefins oligomerization [5-9] over different solid catalysts. As for 1octene oligomerization only a few works are available in the open literature. De Klerk [10] evaluated the liquid-phase oligomerization of 1-octene in the temperature range 373-573K over some solid acid catalysts, such as solid phosphoric acid, amorphous silica, sulfated zirconia, MCM-41 and some zeolites. He found that between $373 \mathrm{~K}$ and $473 \mathrm{~K}$ the main products were $\mathrm{C}_{16}$ dimers but deactivation occurred rapid. When temperature exceeded $473 \mathrm{~K}$ cracking increased considerably and, conversely, the catalyst stability also increased. In another work, de Klerk [11] studied the reactivity differences between 1-octene and 2,4,4-trimethylpentene over solid phosphoric acid in the temperature range of $348-493 \mathrm{~K}$. The observed higher oligomerization, skeletal isomerization and cracking reactivity of the branched olefin compared to 1-octene is explained by the classic carbocation mechanism. De Klerk compared these results with the ion-exchange resin Amberlyst 15, concluding that the latter was more active in the temperature range studied. Grigor'eva et al. [12] studied the oligomerization of 1-octene over several zeolites in the temperature range $423-523 \mathrm{~K}$. They found high activity at temperatures higher than $453 \mathrm{~K}$, being $\mathrm{C}_{16}$ dimers the main product. The concentration 
and strength of the Brönsted acid sites of the zeolites, and the size of their cavities and channels were the most important structural parameters for the oligomerization reaction. Since linear olefins, and in particular 1-octene, are an important feedstock for chemical industry, such as production of Linear Low Density Polyethylene (LLDPE), its production is widely studied [13].

The use of ion-exchange resins as catalysts offers some advantages in the petrochemical industry, such as reduction of equipment corrosion, ease of product separation and catalyst recovering, among others. Ion exchange resins are known to catalyze the oligomerization of olefins, e.g. oligomerization of $\mathrm{C}_{4}$ alkenes over Amberlyst type resins and oligomerization of higher olefins over Nafion resin [14]. Furthermore, ionexchange resins show acid strengths similar to zeolites [15] but higher acid capacity, so they could be used at lower temperatures, limiting side reactions. But surprisingly, there is a lack of works concerning heavy olefins oligomerization using these catalysts.

The aim of this work is to study the liquid-phase conversion of 1-octene over several acidic resins to check the potential of these solids as catalysts in the formation of heavier compounds such as dimers and oligomers. Only macroreticular acidic resins were considered to ensure enough accessibility to acidic centers in the non-polar media due to their permanent porosity. The effect of the temperature on products distribution was also studied. Finally a kinetic model was proposed.

\section{Materials and methods}

\subsection{Chemicals}

1-octene of commercial grade $(97 \%$, Fluka) was used as received in the oligomerization reaction. 1-octene (99.9\%) and 1-hexadecene (97\%) from Acros, and 2-octene (cis + trans, 98.5\%) from TCI-Europe were used for calibration purposes. 


\subsection{Catalysts}

The catalytic behavior of the following acidic macroreticular resins Amberlyst ${ }^{\mathrm{TM}} 15$-wet (A15), 35-wet (A35), 36 (A36), 46 (A46) and 48 (A48) (Rohm and Haas), and Purolite CT175, CT252, CT275 and CT276 (Purolite) were tested. Their physical properties are summarized in Table 1.

Table 1. Physicochemical properties of the resins

\begin{tabular}{|c|c|c|c|c|c|c|c|c|c|}
\hline Catalyst & Crosslinking & Sulfonation $^{\mathrm{a}}$ & $\begin{array}{c}\text { Acidity } \\
\left(\mathrm{eqH}^{+}\right. \\
\left.\mathbf{k g}^{-1}\right)\end{array}$ & $\begin{array}{l}S_{\text {BET }} \\
\left(\mathrm{m}^{2}\right. \\
\left.\mathrm{g}^{-1}\right)\end{array}$ & $\begin{array}{c}V_{\mathrm{g}}{ }^{\mathrm{b}} \\
\left(\mathrm{cm}^{3}\right. \\
\left.\mathrm{g}^{-1}\right)\end{array}$ & $\begin{array}{r}d_{\text {pore }} \mathrm{c} \\
(\AA)\end{array}$ & $\begin{array}{c}\rho_{\mathrm{s}}^{\mathrm{d}} \\
\left(\mathrm{g} / \mathrm{cm}^{3}\right)\end{array}$ & $\begin{array}{c}\theta^{\circ} \\
(\%)\end{array}$ & $\begin{array}{l}-\Delta H_{a d}^{N H_{3}} \\
(\mathbf{k J} / \mathbf{m o l})\end{array}$ \\
\hline A15 & High & $\mathrm{C}$ & 4.81 & 42 & 0.33 & 314 & 1.4164 & 32 & 111 \\
\hline A35 & High & $\mathrm{O}$ & 5.32 & 29 & 0.21 & 290 & 1.5417 & 24 & 117 \\
\hline A36 & Medium & $\mathrm{O}$ & 5.40 & 21 & 0.14 & 270 & 1.5671 & 18 & 117 \\
\hline A46 & High & SS & 0.43 & 57 & 0.26 & 182 & 1.1369 & 23 & - \\
\hline A48 & High & $\mathrm{O}$ & 5.62 & 34 & 0.25 & 294 & 1.5378 & 28 & - \\
\hline CT175 & High & $\mathrm{C}$ & 4.98 & 29 & 0.48 & 662 & 1.4984 & 42 & 114 \\
\hline CT252 & Medium & $\mathrm{O}$ & 5.40 & 22 & 0.22 & 400 & 1.4929 & 25 & - \\
\hline CT275 & High & $\mathrm{O}$ & 5.20 & 28 & 0.42 & 600 & 1.5056 & 39 & 119 \\
\hline СТ276 & Medium & $\mathrm{O}$ & 5.20 & 24 & 0.21 & 350 & 1.4890 & 24 & - \\
\hline
\end{tabular}

from adsorption/desorption of $\mathrm{N}_{2}$ at $77 \mathrm{~K} .{ }^{\mathrm{c}}$ assuming pore cylindrical model. ${ }^{\mathrm{d}}$ skeletal density, measured by helium displacement. ${ }^{\mathrm{e}}$ porosity was estimated as $100 V_{\mathrm{g}} /\left(V_{\mathrm{g}}+\left(1 / \rho_{\mathrm{s}}\right)\right)$

The adsorption enthalpy of ammonia [16] is included as a relative measure of the acid strength of the ion exchange resins. Amberlyst 15 is conventional sulfonated with a high crosslinking degree. Amberlyst 35 and 36 are oversulfonated with high and medium crosslinking degree, respectively. Both properties have a clear effect on the physical properties of the resins. A decrease of the crosslinking degree leads to a diminution of the surface area, pore volume and porosity, whereas the oversulfonation involves an increase of the acid capacity and acid strength but also of the stiffness of the structure. 
Consequently, the surface area, pore volume and porosity of oversulfonated resins are lower than the analogue conventional sulfonated resins (see Amberlyst 15 and 35). Similarly, CT175 and CT275 differ on the sulfonation degree and acid strength, being both high crosslinked macroreticular resins. CT252 and CT276 are both medium crosslinked and oversulfonated with a wide pore diameter. Amberlyst 46 should be highlighted. It has a very low acidity but it is only surface-sulfonated, which means that all acid centers are fully accessible.

The acidic ion-exchange resins were delivered saturated with water. They were activated by drying at $383 \mathrm{~K}$, first at atmospheric pressure and then overnight under vacuum at the same temperature. Final water content after drying was less than $3 \% \mathrm{w} / \mathrm{w}$ (Karl-Fisher titration). Commercial particles sizes were used in all the experiments.

\subsection{Analysis}

All samples were analyzed by gas chromatography. Liquid samples were obtained through a sampling valve (Valco A2CI4WE.2) that injected $0.2 \mu \mathrm{L}$ of pressurized liquid into a Hewlett-Packard 6890 gas chromatograph equipped with a capillary column (HP 190915-433; 5\% phenyl methyl siloxane, $30 \mathrm{~m} \times 250 \mu \mathrm{m} \times 0.25 \mu \mathrm{m}$ nominal). A mass selective detector (HP 5973) was used in the gas chromatography (GC) analysis. The injector temperature was set to $523 \mathrm{~K}$, the electron source of the mass detector was set to $503 \mathrm{~K}$, and the quadrupole was set to $423 \mathrm{~K}$. A temperature ramp was programmed: from $308 \mathrm{~K}$ (isotherm during $1.5 \mathrm{~min}$ ) to $473 \mathrm{~K}$ at a $50 \mathrm{~K} \cdot \mathrm{min}^{-1}$ ramp rate, with the final temperature maintained for 10 min. Helium (Air Liquide, Barcelona, Spain) with a minimum purity of $99.998 \%$ was used as a carrier gas.

\subsection{Apparatus and procedure}


Experiments were carried out in a $200-\mathrm{cm}^{3}$ stainless steel jacketed batch reactor (Autoclave Engineers). The stirring speed was set to $500 \mathrm{rpm}$, in order to avoid external mass transfer effects [17] and the temperature range was set to $353-393 \mathrm{~K}$, which was controlled by a thermostated 1,2-propanediol-water mixture within $\pm 0.1 \mathrm{~K}$. The reaction pressure was maintained with nitrogen at $2 \mathrm{MPa}$ to ensure the liquid phase for the reacting system.

The reactor was loaded with $4 \mathrm{~g}$ of fresh dried resin and, after the desired temperature was reached, pure 1-octene was shifted to it by means of nitrogen from a calibrated burette. No solvents were used. Then, agitation was switched on and this moment was considered as the starting point of the reaction. Periodically, liquid samples were taken out to be analyzed. Experiments lasted 6 hours. Deactivation of the catalyst can be discarded in this work. More detailed information about the equipment can be found elsewhere [17].

\subsection{Calculations}

For each experiment, 1-octene conversion $\left(X_{1-\text { oct }}\right)$, selectivity to linear isomers $\left(S_{\text {linearC8 }}\right)$, selectivity to branched isomers $\left(S_{\text {branchedC8}}\right)$ and selectivity to dimers $\left(S_{C 16}\right)$ were calculated using the following expressions:

$$
\begin{aligned}
& X_{1 \text { oct }}=\frac{n_{\text {loct }}^{0}-n_{\text {loct }}}{n_{\text {loct }}^{0}}=\left[\frac{n_{\text {linearC8 }}+n_{\text {branchedC8 }}+2 n_{C 16}}{n_{\text {loct }}+n_{\text {linearC } 8}+n_{\text {branched } C 8}+2 n_{C 16}}\right]_{t} \\
& S_{\text {linearC8 }}=\left[\frac{n_{\text {linearC8 }}}{n_{\text {linearC8 }}+n_{\text {branched } C 8}+2 n_{C 16}}\right]_{t} \\
& S_{\text {branchedC8 }}=\left[\frac{n_{\text {branchedC8 }}}{n_{\text {linearC8 }}+n_{\text {branched } C 8}+2 n_{C 16}}\right]_{t}
\end{aligned}
$$




$$
\begin{aligned}
& S_{C 16}=\left[\frac{2 n_{C 16}}{n_{\text {linearC8 }}+n_{\text {branched } 8}+2 n_{C 16}}\right]_{t} \\
& -r_{1-\text { octene }}^{0}=\frac{1}{W}\left[\frac{d n_{1-\text { octene }}}{d t}\right]_{t=0} \\
& - \text { TOF }^{0}=\frac{r_{1-\text { octene }}^{0}}{\text { acidity }}
\end{aligned}
$$

Each family of species of linear $\mathrm{C}_{8}$ isomers, branched $\mathrm{C}_{8}$ isomers and $\mathrm{C}_{16}$ dimers were lumped together for the calculations. Replicated runs were made for some crucial experiments and the reproducibility of the results was found to be reliable. The standard deviation for 1 -octene conversion was $1.6 \%$ and $2 \%$ for selectivity. On the other hand, the error of the mass balances was $<7 \%$ for all the experiments.

\section{Results and Discussion}

\subsection{Reaction network}

Figure 1 shows the mole profile of an experiment performed at $373 \mathrm{~K}$ on Amberlyst 35. Similar profiles were obtained with the rest of the resins. 1-octene was rapidly consumed to form linear $\mathrm{C}_{8}$ isomers. Among them, 2-octene was the main product, although 3-octene and 4-octene were also produced. These reaction products were also found by de Klerk [11] over solid phosphoric acid and Grigor'eva [12] over a zeolite Y. $\mathrm{C}_{8}$ linear isomers mole profile increased at the beginning of the reaction, passed through a maximum, and then decreased as it was consumed to form $\mathrm{C}_{8}$ branched isomers and $\mathrm{C}_{16}$ dimers. Both $\mathrm{C}_{8}$ branched isomers and $\mathrm{C}_{16}$ dimers increased smoothly during the runs. 


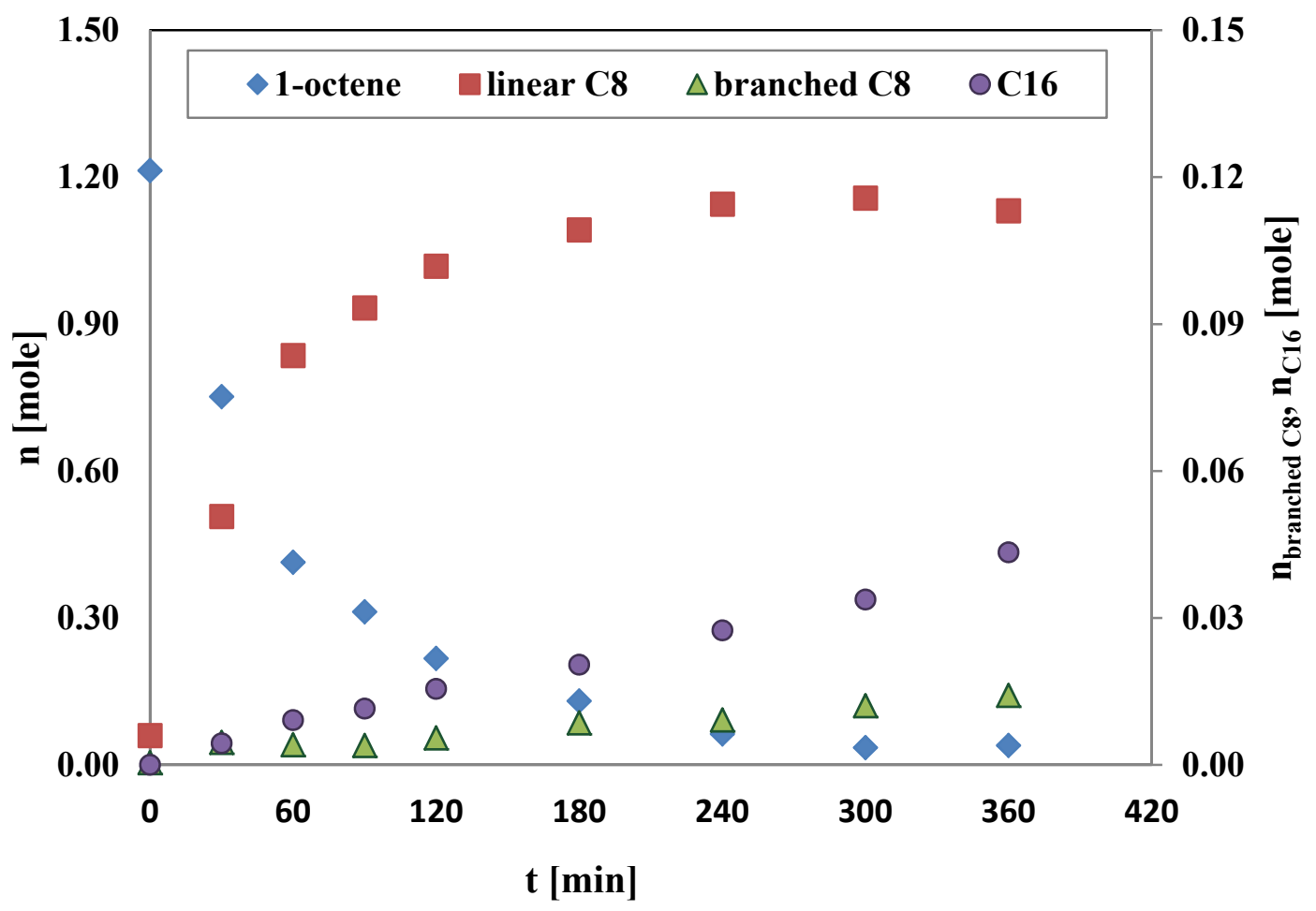

Figure 1. Mole profile of a model experiment over $4 \mathrm{~g}$ A35 at $373 \mathrm{~K}$ and $2 \mathrm{MPa}$

$\mathrm{C}_{24}$ trimers and heavier oligomers were not chromatographically detected at the temperature range explored after $6 \mathrm{~h}$ of reaction. As these heavy compounds are difficult to be properly determined by the analytical method above mentioned, simulated distillation analysis of the product mixture based on the ASTM Standards Test Method D-2887 were performed. As Figure 2 shows, all the hydrocarbons of the mixture distillated at temperatures lower than $600 \mathrm{~K}$. Boiling points of $\mathrm{C}_{8}$ and $\mathrm{C}_{16}$ alkenes are around $400 \mathrm{~K}$ and $560 \mathrm{~K}$, respectively, therefore heavier oligomers than $\mathrm{C}_{16}$ can be discarded. 


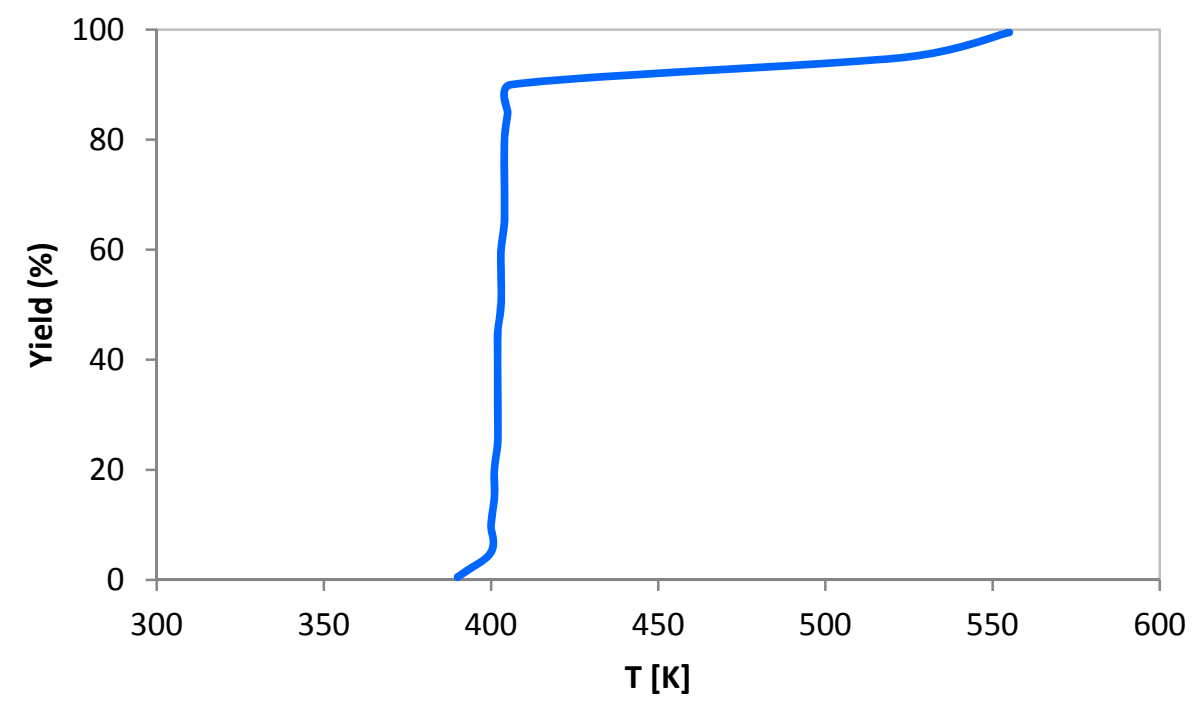

Figure 2. Simulated distillation curve of the hydrocarbon mixtures obtained from 1octene oligomerization over Amberlyst 35 at $373 \mathrm{~K}$ after $6 \mathrm{~h}$ of reaction

Branched $\mathrm{C}_{8}$ isomers were detected in less extent (less than $4 \% \mathrm{w} / \mathrm{w}$ ). Among these products, 3-methyl-1-heptene, 3-ethyl-2-hexene, 5-methyl-1-heptene and 3-methyl-2heptene were identified by the library of the MS software with a very reasonable confidence. No aromatics and cracking products were detected in any of the runs performed at $373 \mathrm{~K}$. De Klerk [10] suggested that double bond isomerization is the main reaction at low temperatures and cracking and hydrogen transfer products become important at temperatures higher than $473 \mathrm{~K}$ for 1-octene oligomerization. Also, he announced that $\mathrm{C}_{8}$ isomers are susceptible to cracking themselves, so this reaction and the oligomerization proceed in parallel. From our results it is inferred that higher temperatures than $373 \mathrm{~K}$ are needed to observe $\mathrm{C}_{8}$ cracking over ion-exchange resins. Schwarzer et al. [5] reported the effect of solid phosphoric acid (SPA) acid strength on products distribution for 1-hexene dimerization. They suggested that, at lower acid strength, after a fast double bond shift isomerization, linear hexenes were directly oligomerizated and, at high SPA acid strength, linear hexenes were first isomerizated to skeletal hexene isomers before dimerization. From our results, it can be assumed that 
acidic ion-exchange resins of present work have enough acid strength to catalyze double-bond isomerization, some oligomerization for 1-octene and skeletal isomerization in low extent but not enough to catalyze disproportionation and cracking. Figure 3 proposes a reaction network for 1-octene dimerization, which agrees with that proposed by de Klerk [10,11]. Also, it is similar to that found for 1-hexene oligomerization $[4,6]$ in the open literature. It is possible to compare 1-octene oligomerization results with 1-hexene oligomerization [15] performed in the same setup under similar conditions. At $373 \mathrm{~K}$ we found that Amberlyst 35 was active enough to produce up to $\mathrm{C}_{18}$ oligomers, but, again, neither cracking products nor skeletal isomerization products were obtained. Comparing both reaction systems at $373 \mathrm{~K}$ using Amberlyst 35 as catalyst, it can be inferred that the reactivity of 1-octene is lower than 1-hexene, which agrees with what suggested de Klerk [11].

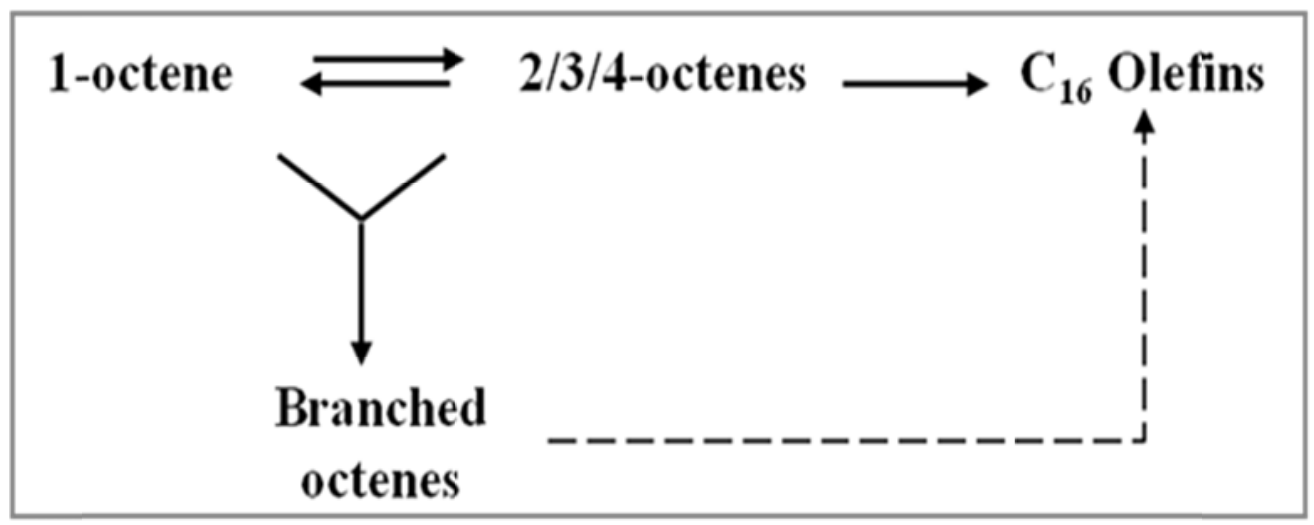

Figure 3. Reaction network proposed for 1-octene dimerization

\subsection{Effect of the catalyst structure on the catalytic results}

Figure 4 (up and down) shows the evolution of 1-octene conversion along time for all ion-exchange resins tested. 

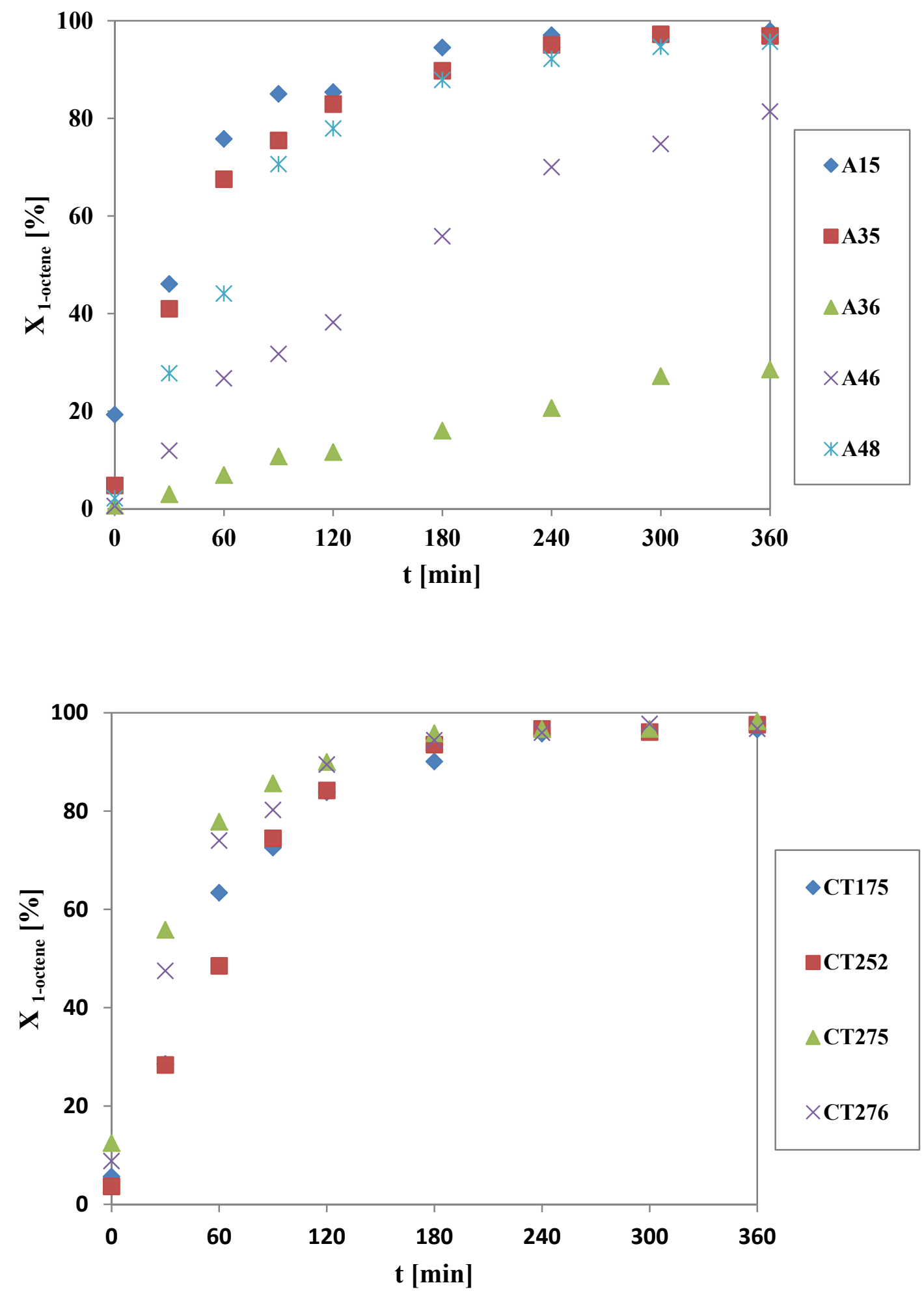

Figure 4. Conversion of 1 -octene at $373 \mathrm{~K}$ for macroreticular Amberlyst resins (up) and Purolite resins (down) over $4 \mathrm{~g}$ of catalyst and $2 \mathrm{MPa}$. 
Similar results were obtained for all catalysts except Amberlyst 46 and 36. At the beginning of the experiment, a sharp increase on the profile is observed, meaning high reaction rates. After 3 hours, 1-octene conversion was around $90 \%$ over most catalysts, reaching a value around $97 \%$ at the end of the runs. The most active tested resins at the end of the runs were oversulfonated (Amberlyst 35 and 48, CT252, CT275, CT276), what indicates that acid capacity is a relevant resin property to convert 1 -octene. The conventionally sulfonated resins Amberlyst 15 and CT175 were slightly less active. The lower activity of Amberlyst 46 can be explained by the fact that it has an acid capacity ten-fold lower than the other catalysts.

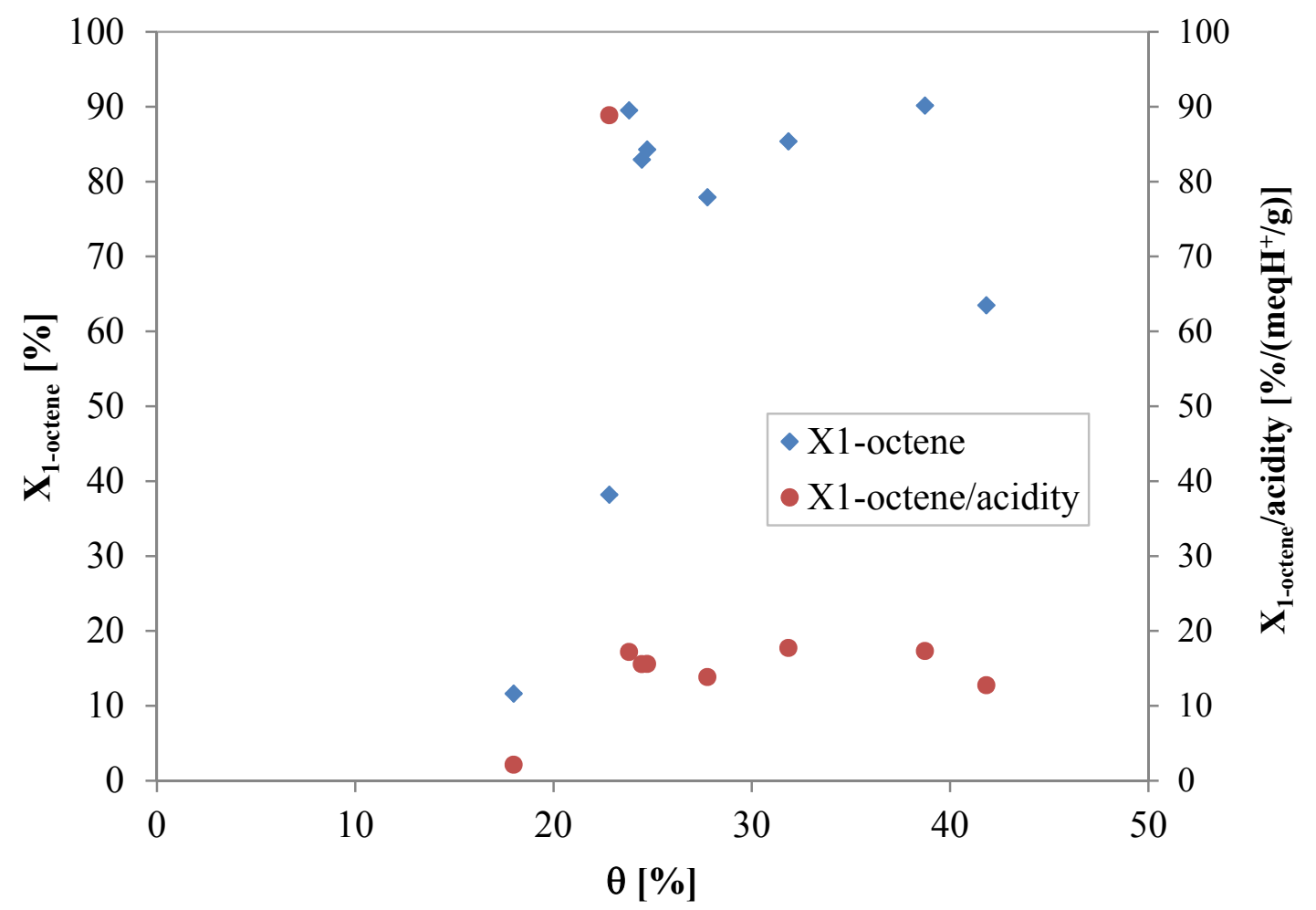

Figure 5. Effect of porosity on 1-octene conversion and modified 1-octene conversion for all catalysts at $373 \mathrm{~K}$ and $2 \mathrm{MPa}$ after $2 \mathrm{~h}$ of run.

As for Amberlyst 36 (the experiment was replicated twice to confirm results), although it has a very high acid capacity, the activity shown is quite low. As the medium is clearly non-polar, its structure is collapsed. This point is confirmed by its low porosity 
(18\%, see Table 1 and Figure 5). Consequently, only a reduced fraction of the active centers is accessible for 1-octene. In fact, when comparing the behavior of all resins with Amberlyst 46 (low acid capacity but all acid sites are accessible since it is only surface-sulfonated), it can be inferred that only a part of the active sites of the rest of the catalysts is accessible, mainly those located at the more external layers of the gel-phase of their structure. This is clearly observed when plotting 1-octene conversion modified by the acid capacity $\left(\mathrm{X}_{1-\text { octene }} /\right.$ acidity) versus porosity (see Figure 5 ). Figure 5 plots conversion after $2 \mathrm{~h}$ and conversion modified by the acid capacity because at the end of the experiment most of the catalysts reach quasi complete conversion. The point with a conversion per active site of 90 corresponds to Amberlyst 46, while the other catalysts have values lower than 20 with the exception of Amberlyst 36 that has a value of 2 . This better behavior of Amberlyst 46 per active site is observed during the whole experiment, as it is shown in Figure 6. When plotting 1-octene conversion versus the contact time per active site $\left(t \cdot W \cdot\left(n_{1-o c t e n e}^{0}\right)^{-1} \cdot\right.$ acidity, where $W$ is the mass of catalyst $)$, the catalysts can be grouped into three groups. Firstly, Amberlyst 46 shows the best performance per active site, as it reached higher conversion values at lower contact time. The second group is formed by all other resins except Amberlyst 36, represented in Figure 6 only by Amberlyst 35 and CT275 for the sake of clarity. Among this group some small differences can be observed, e.g. CT275 seems more active per active center than Amberlyst 35. Finally, the third group is formed by Amberlyst 36, which showed an activity much lower than the others, as commented before. 


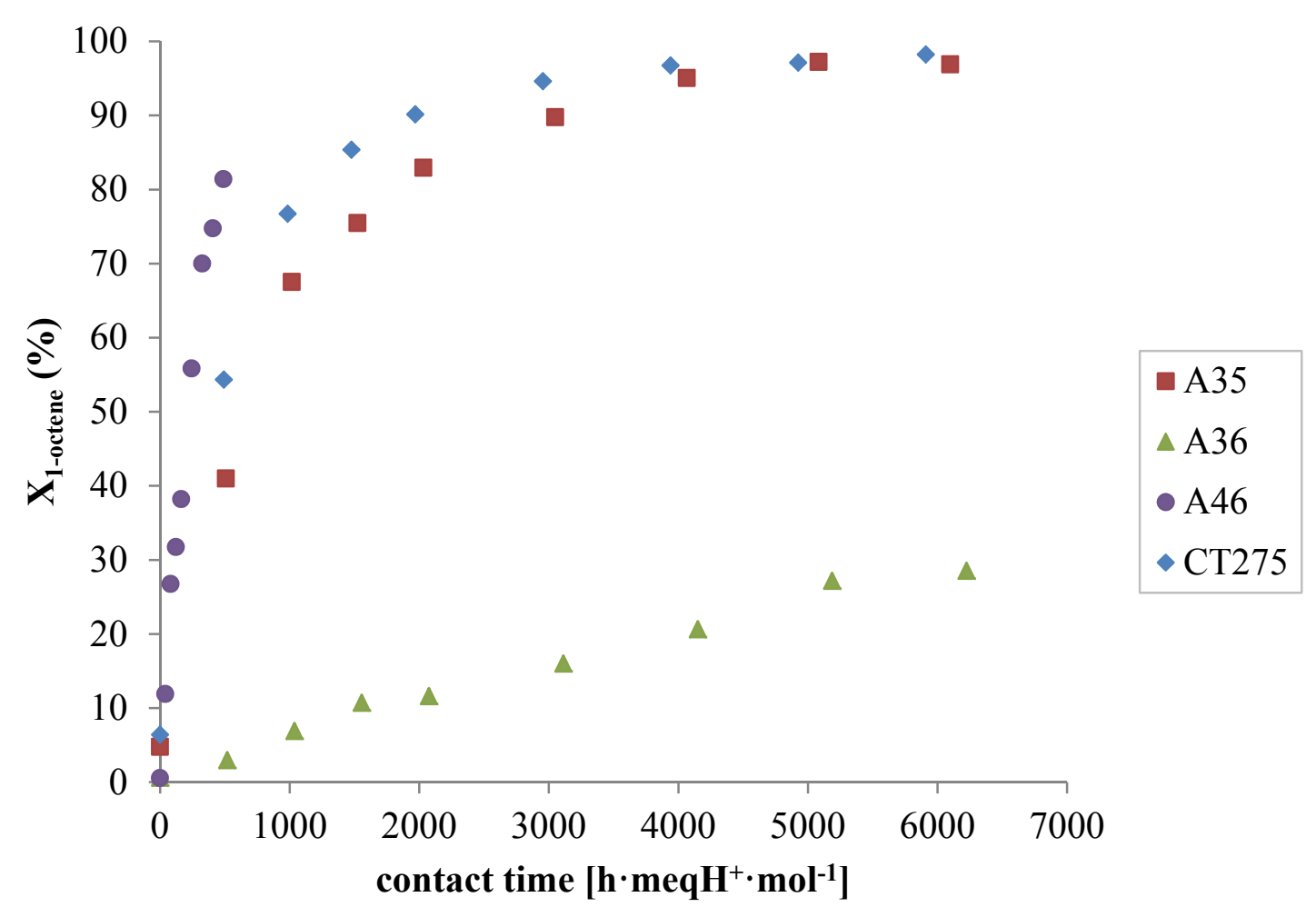

Figure 6. 1-octene conversion versus contact time for Amberlyst 35, 36, 46 and CT275. $\mathrm{T}=373 \mathrm{~K}, 4 \mathrm{~g}$ catalyst, $2 \mathrm{MPa}$.

If we assume that Amberlyst 46 has all active sites accessible and these are of the same nature for all catalysts, by considering TOF values at time 0 (equation 6 ), only $22-48 \%$ of the active sites of the other catalysts would be involved in the reaction, $1 \%$ in the case of Amberlyst 36 (see Table 2). From these results, an estimation of the accessible acidity can be computed, as shown in Table 2 .

Table 2. Accessible acidity for all catalysts considering TOF values at $\mathrm{t}=0$ at $373 \mathrm{~K}$ and Amberlyst 46 as a reference.

\begin{tabular}{ccccc}
\hline & $\begin{array}{c}\mathrm{r}_{1-\mathrm{octene}} \mathrm{o}^{-1} \\
\left(\mathrm{~mol} \cdot \mathrm{min}^{-1} \cdot \mathrm{kg}^{-1}\right)\end{array}$ & $\begin{array}{c}\mathrm{TOF}^{0} \\
\left(\mathrm{~mol} \cdot \mathrm{min}^{-1} \cdot \mathrm{eqH}^{-1}\right)\end{array}$ & $\begin{array}{c}\text { Accessible active sites } \\
(\%)\end{array}$ & $\begin{array}{c}\text { Accessible acidity } \\
\left(\mathrm{meqH} \cdot \mathrm{g}^{-1}\right)\end{array}$ \\
\hline A15 & 9.1 & 1.9 & 32 & 1.52 \\
A35 & 10.0 & 1.9 & 31 & 1.66 \\
A36 & 0.3 & 0.1 & 1 & 0.06 \\
A46 & 2.6 & 6.0 & 100 & 0.43 \\
A48 & 7.4 & 1.3 & 22 & 1.23 \\
CT175 & 8.8 & 1.8 & 29 & 1.46 \\
CT252 & 8.5 & 1.6 & 26 & 1.42 \\
CT275 & 15.0 & 2.9 & 48 & 2.49
\end{tabular}


Figure 7 plots the selectivity of the reaction after $6 \mathrm{~h}$ at $373 \mathrm{~K}$. As mentioned above, the main reaction was the double bond isomerization to form linear $\mathrm{C}_{8}$ alkenes. The skeletal isomerization was also observed on all catalysts, but in very low extent (less than $2 \%$ ). Dimers were formed with all the resins, except for Amberlyst 36. Selectivity to dimers after $6 \mathrm{~h}$ of reaction was between 6 and 12\%, depending on the used resin. The highest value was obtained on CT275 (12\%). Again, oversulfonated resins showed a higher activity to dimerization than conventional ones, confirming the important effect of the acidity and acid strength when the acid sites are accessible.

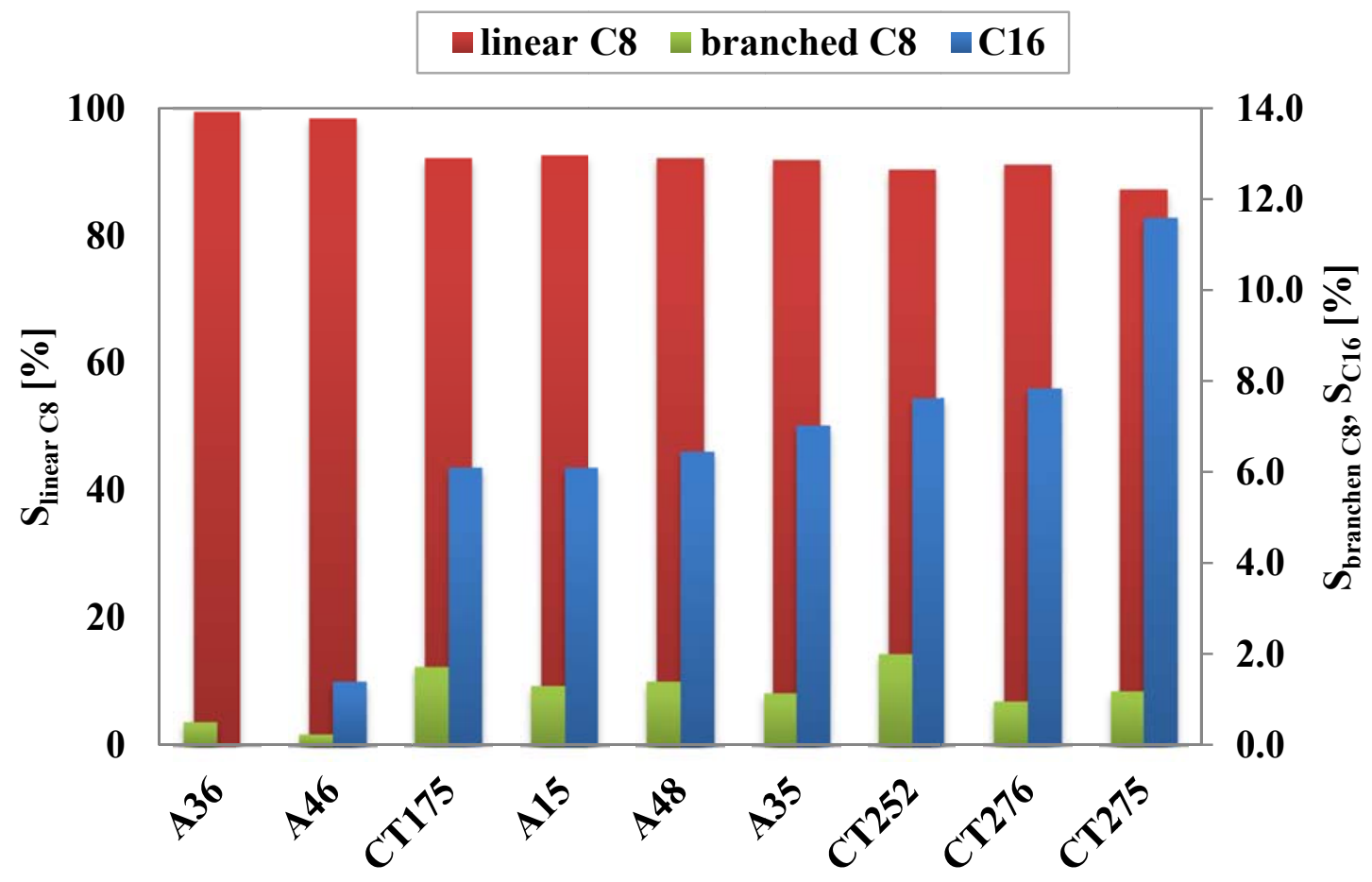

Figure 7. Selectivity to linear $\mathrm{C}_{8}$ isomers, branched $\mathrm{C}_{8}$ isomers and dimers at $373 \mathrm{~K}$ after $6 \mathrm{~h}$ over $4 \mathrm{~g}$ of catalyst and $2 \mathrm{MPa}$. 
Figure 8 depicts the selectivity after 6 h versus the accessible acidity computed above, assuming that this accessible acidity does not change as reaction proceeds. Although this factor does not take into account the difference of active sites among catalysts, e.g. different acid strength, Figure 8 shows a clear dependence between selectivity and the accessible acidity, and it permits explaining the obtained results from a semiempirical point of view.

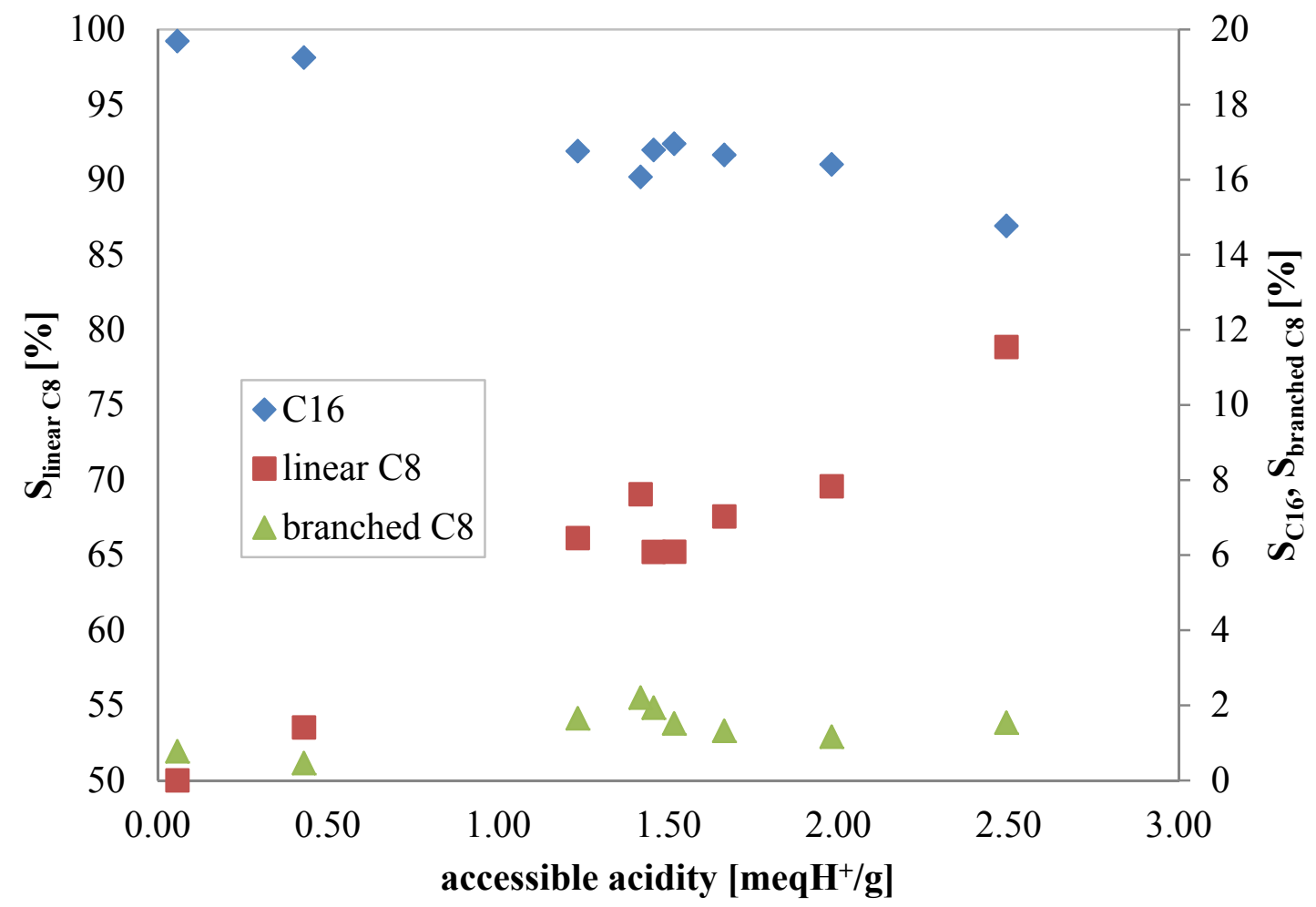

Figure 8 . Selectivity of the reactions after $2 \mathrm{~h}$ at $373 \mathrm{~K}$ versus accessible acidity over $4 \mathrm{~g}$ of catalyst and $2 \mathrm{MPa}$.

\subsection{Effect of temperature}

The temperature effect was tested on Amberlyst 35 (353 - 393K) and on CT275 (353$383 \mathrm{~K})$. These catalysts were chosen because they were two of the best at $373 \mathrm{~K}$, and they are extensively used in industry. As expected, temperature has an important effect 
on the activity, resulting in an increase of 1-octene conversion at the beginning of the reaction at higher temperatures (see Figure 9). At the lowest temperature checked, after $6 \mathrm{~h}$ only $60 \%$ conversion on Amberlyst 35 was achieved. Similar results were obtained on CT275.

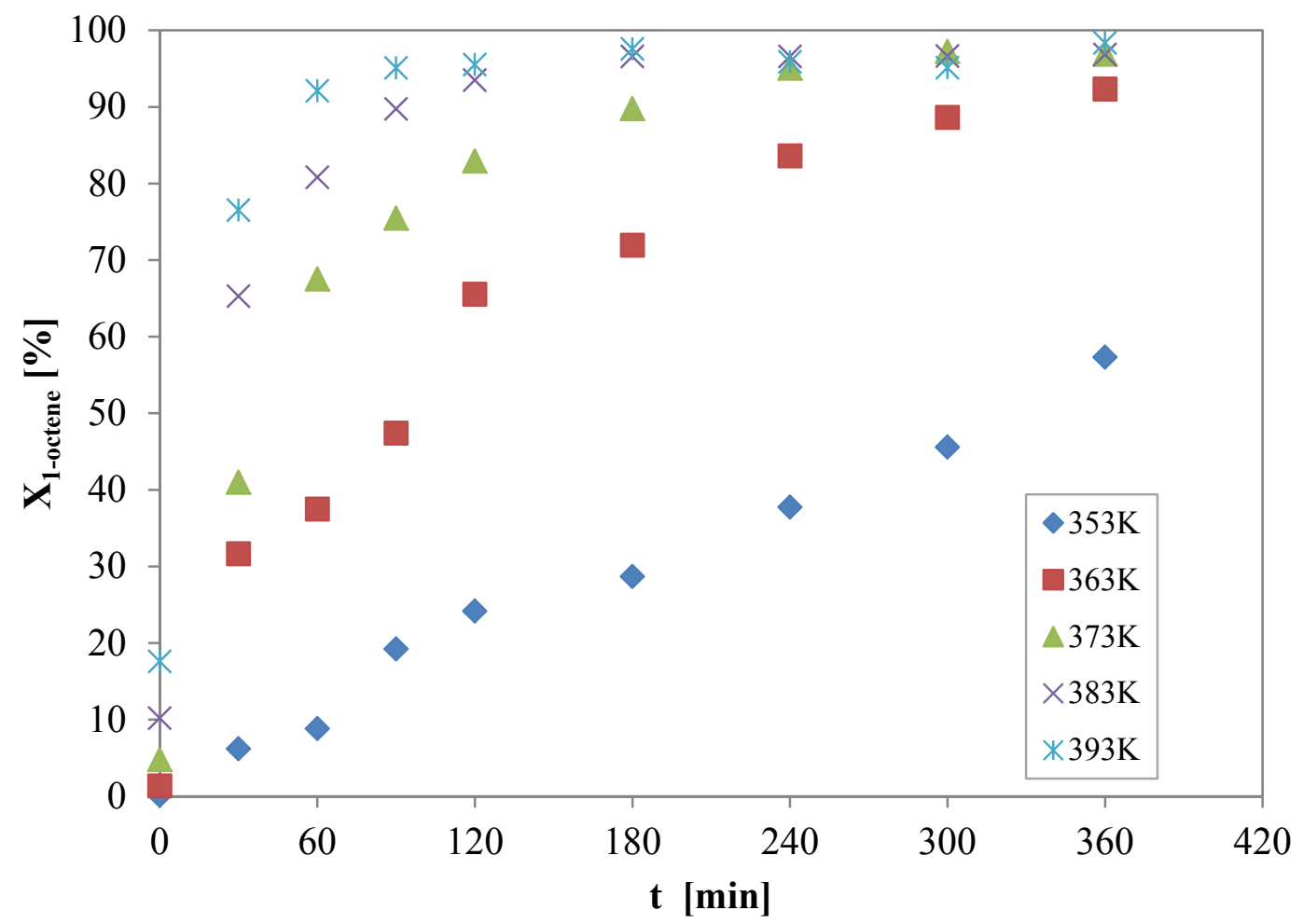

Figure 9. Evolution of 1-octene conversion over time at different temperatures on $4 \mathrm{~g}$ of Amberlyst 35 and 2MPa.

The temperature had a positive effect on the selectivity to $\mathrm{C}_{16}$ products. At the lowest temperature only the double bond isomerization took place on Amberlyst 35 and a little dimerization on CT275 (see Figure 10). On increasing the temperature, the skeletal isomerization and mainly the dimerization reactions became more important, reaching a selectivity value to $\mathrm{C}_{16}$ products of $40 \%$ at $393 \mathrm{~K}$ on Amberlyst 35 . Surprisingly, neither heavier oligomers nor cracking products were detected in the temperature range explored. 1-hexene oligomerization performed in the same setup [15] showed a very 
high selectivity to $\mathrm{C}_{12}$ dimers $(>70 \%)$ and the formation of $\mathrm{C}_{18}$ trimers and cracking products at temperatures higher than $383 \mathrm{~K}$. Probably higher temperatures are needed to observe trimerization of 1-octene on acidic ion-exchange resins.

The behavior of both catalysts was similar at all temperatures, being CT275 slightly more active to dimerization than Amberlyst 35.

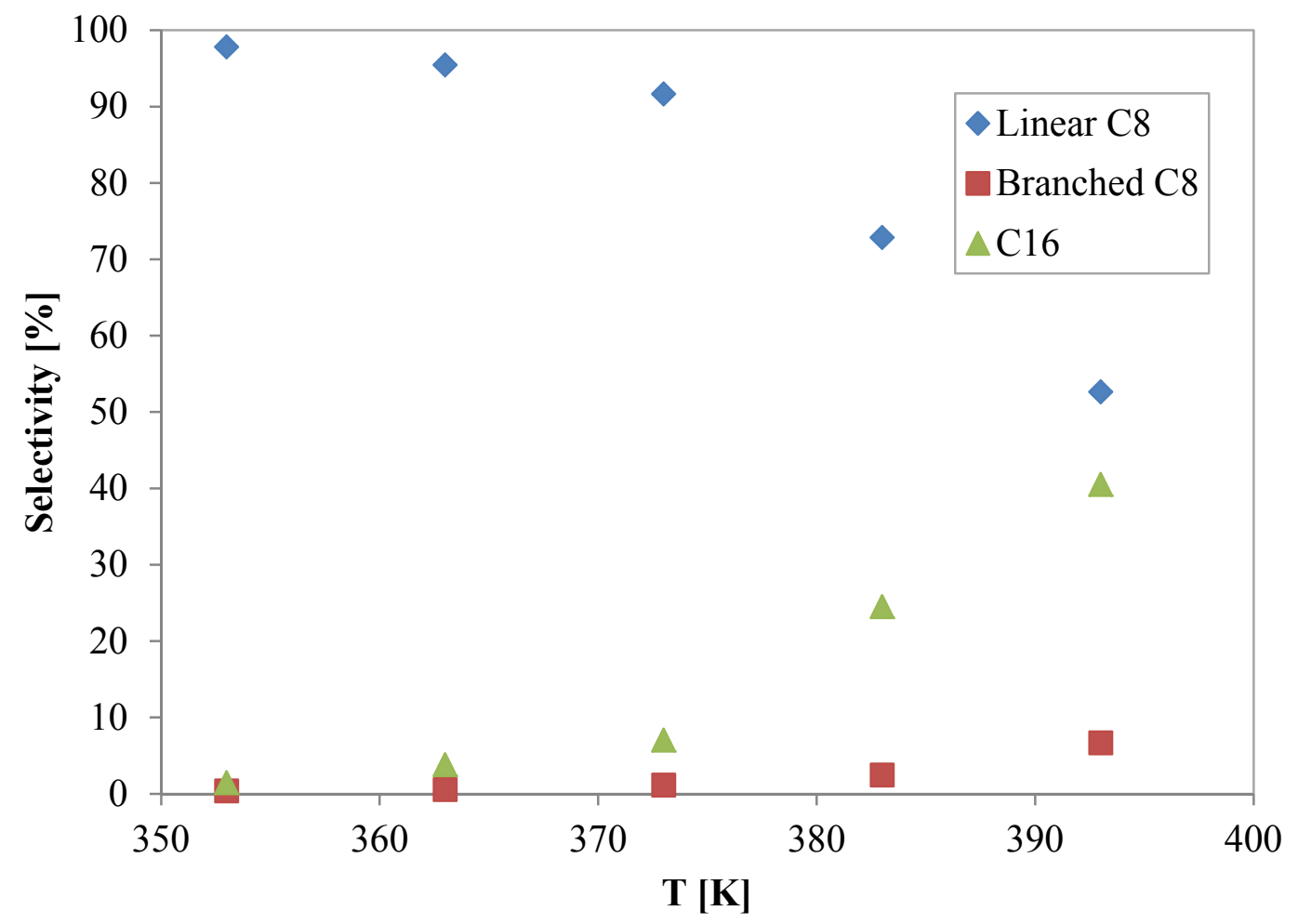




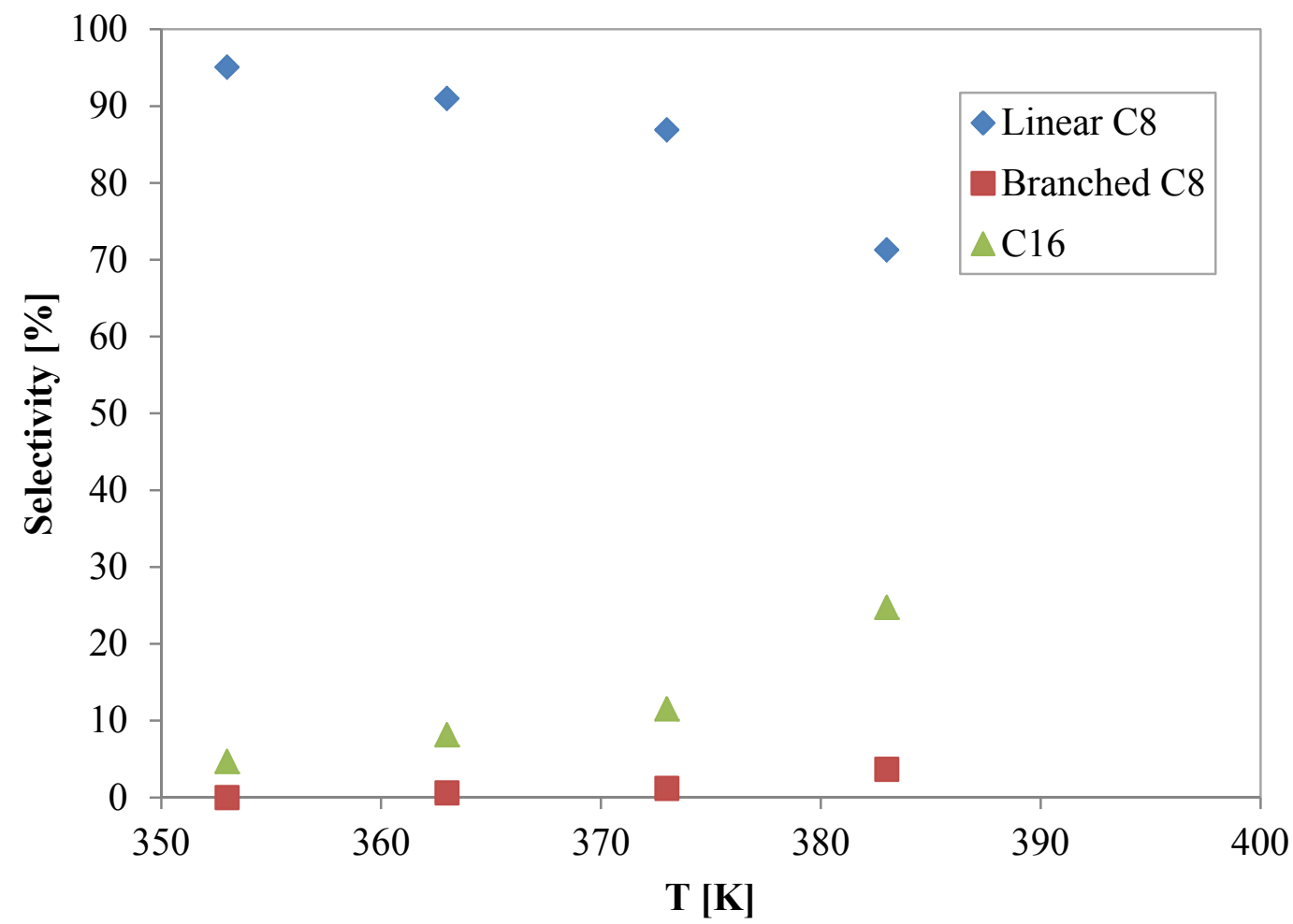

Figure 10. Evolution of the selectivity of the reaction with temperature on Amberlyst 35 (up) and CT275 (down) on $4 \mathrm{~g}$ of catalyst and $2 \mathrm{MPa}$

\subsection{Kinetic study}

Due to the large amount of formed isomers and that they cannot be individually quantified through the chromatographic analysis, different compounds families have been lumped together with the aim of obtaining a kinetic description for the reacting system. Compounds have been grouped as 1-octene (compound A), linear isomers (B), branched $\mathrm{C}_{8}$ isomers $(\mathrm{C})$ and $\mathrm{C}_{16}$ dimers $(\mathrm{D})$. Accordingly to the proposed reaction network (Figure 3), and with the assumption that each reaction is elementary, the following reactions and kinetic expressions for the respective reaction rates have been considered:

$$
A \stackrel{I}{\rightleftharpoons} B \quad r_{I}=k_{I}\left(c_{A}-c_{B} / K_{e q, I}\right)
$$




$$
\begin{array}{ll}
B \stackrel{I I}{\rightleftharpoons} C & r_{I I}=k_{I I}\left(c_{A}-c_{B} / K_{\text {eq,II }}\right) \\
2 B \stackrel{I I I}{\longrightarrow} D & r_{I I I}=k_{I I I} c_{B}^{2}
\end{array}
$$

In this reaction scheme, 1-octene $(\mathrm{A})$ is considered to be involved only in a reversible reaction, as it is rapidly consumed, to form linear $\mathrm{C}_{8}$ isomers (reaction $\mathrm{I}$ ). They can undergo to form branched $\mathrm{C}_{8}$ isomers via skeletal isomerization (reaction II) or dimerize to form $\mathrm{C}_{16}$ products (reaction III). In the reaction rate equations, $k_{I}, k_{I I}$ and $k_{I I I}$ are the kinetic constants for the respective reactions. Reactions I and II are considered to be equilibrium reactions, with equilibrium constants $K_{\text {eq,i, }}$, and dimerization reaction III is non-reversible, accordingly to the experimentally observed evolution of chemical species. Dimerization reaction of branched isomers $\mathrm{C}$ has not been taken into account due to their low content in the reaction medium, and to avoid an excess of the kinetic constants to be fitted that would result in a higher constant correlation in their determination and, therefore, a larger estimation error. In the stirred batch reactor, mass balances for the different species are determined by the stochiometric scheme:

$$
\begin{aligned}
& \frac{d n_{A}}{d t}=-W r_{I} \\
& \frac{d n_{B}}{d t}=W\left(r_{I}-r_{I I}-2 r_{I I I}\right) \\
& \frac{d n_{C}}{d t}=W r_{I I} \\
& \frac{d n_{D}}{d t}=W r_{I I I}
\end{aligned}
$$

Where $n_{i}$ is mole of species $i$ and $r_{j}$ is the intrinsic reaction rate for reaction $j$.

The simultaneous combination of equations 5 to 11 results in a set of differential equations, whose integration with the appropriate values of the kinetic and equilibrium constants gives a prediction of the evolution of the different species content in the 
reaction medium. To solve this set of equations, which could become a stiff problem depending on the constant values, a mixed Adams-Backward Differentiation Formulas (BDF) time integration method that automatically selects between nonstiff (Adams) and stiff (BDF) has been used in a tailor-made computer program. Optimization routines based on the Levenberg-Marquardt method have been also implemented in the code to obtain the best set of kinetic and equilibrium constant values that describe satisfactorily the experimental data. The objective function for optimization has been the weighted sum of squares of the deviations between experimental mole and calculated mole at different reaction times, the weighting factor being the mean mole content of each species during each experiment to obtain a good simultaneous fit of all species independently of their content. For mathematical reasons, to get a better convergence for the optimization method and a normally distributed parameter estimation, fitted parameters are not the kinetic and equilibrium constants themselves, but the following exponents $k_{i}$ :

$$
k_{I}, K_{\text {eq, }}, k_{I I}, K_{e q, I I}, k_{I I I}=\exp \left(k_{i}\right), \text { for } i=1 \text { to } 5 \text {, respectively }
$$

In addition, the standard error of each fitted parameter has been estimated from the diagonal elements of the parameter covariance matrix.

In a first step, the experiments performed at $373 \mathrm{~K}$ have been considered, separately grouped according to the used catalyst, and the optimum set of parameters $k_{1}$ to $k_{5}$ has been obtained for each catalyst. For most of the catalysts, the predicted mole evolution fitted satisfactorily the experimental values, although a slight discrepancy is observed for $\mathrm{C}_{16}$ compounds, what is attributed to the fact that they group a large number of species. As example, the results for CT252 are shown in Figure 11, similar to most of the experiments, except for A36 and A46. For these two resins, the shape of the 
predicted evolution differs from the experimental shape, and, as they seem to follow a different kinetics, they have not been included in the further analysis of the results. In fact, both resins present different properties to the rest of the catalysts, as previously commented, and A36 was the only resin where dimerization did not take place.

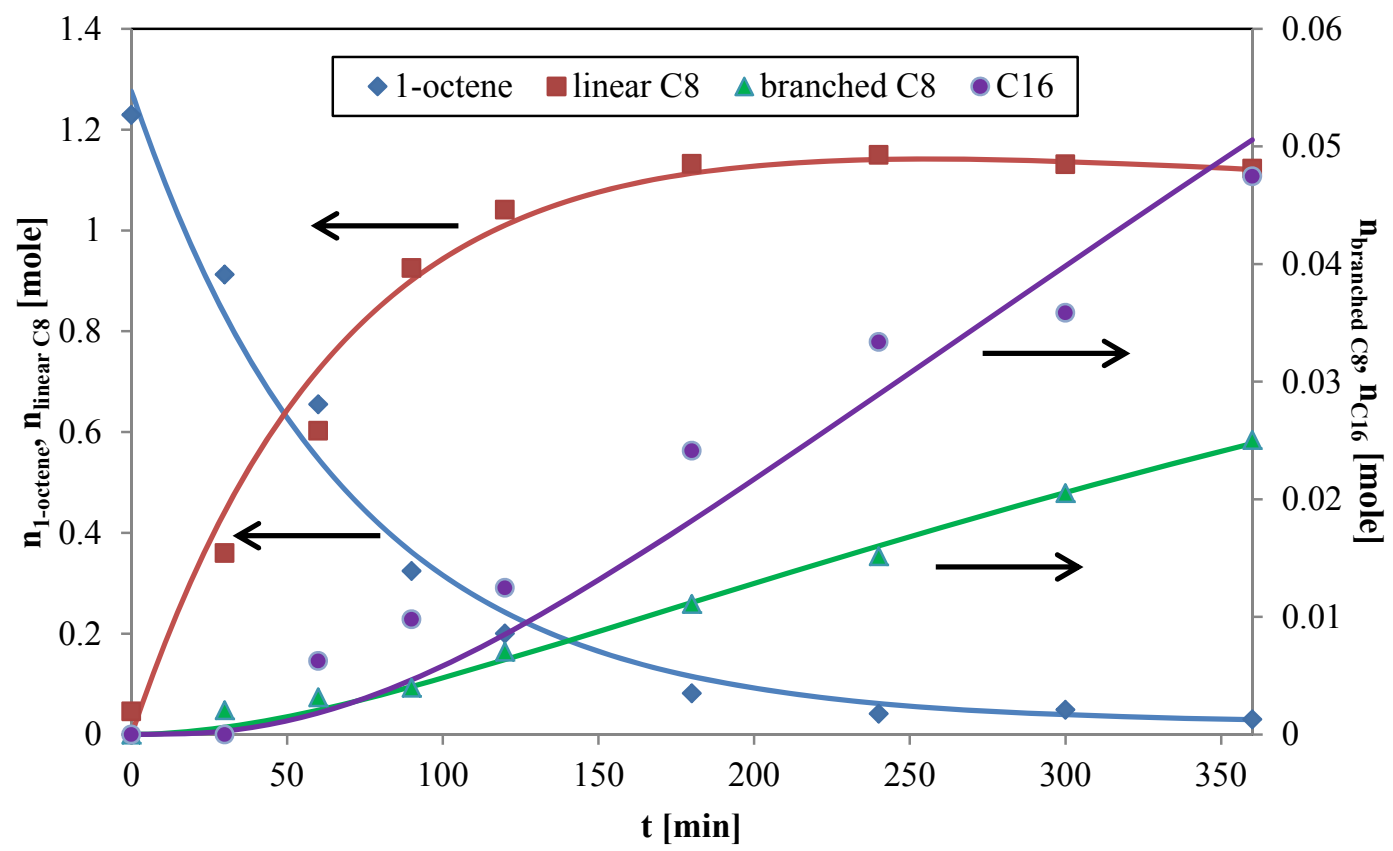

Figure 11: Reaction progress of the simultaneous isomerization and dimerization of 1-octene for resin CT252 at $373 \mathrm{~K}$. Points are experimental values, and solid lines are the predicted evolution by using the optimum fitted parameter values.

With respect to the five fitted parameters for each resin, two of them, $k_{2}$ and $k_{4}$, are related to the equilibrium constants (see Equation 14), and therefore they should be similar for all resins. As it can be seen in Figure 12, they are indeed statistically equal. It is worth noting that these values were obtained from experiments where equilibrium was not completely reached, as reaction III (Eq. 7) is not a reversible reaction, but the same value for all resins was obtained. The rest of the parameters $\left(k_{1}, k_{3}\right.$ and $\left.k_{5}\right)$ are related to the kinetic constants and, as a consequence, to the catalyst activity, that differs for each resin. 


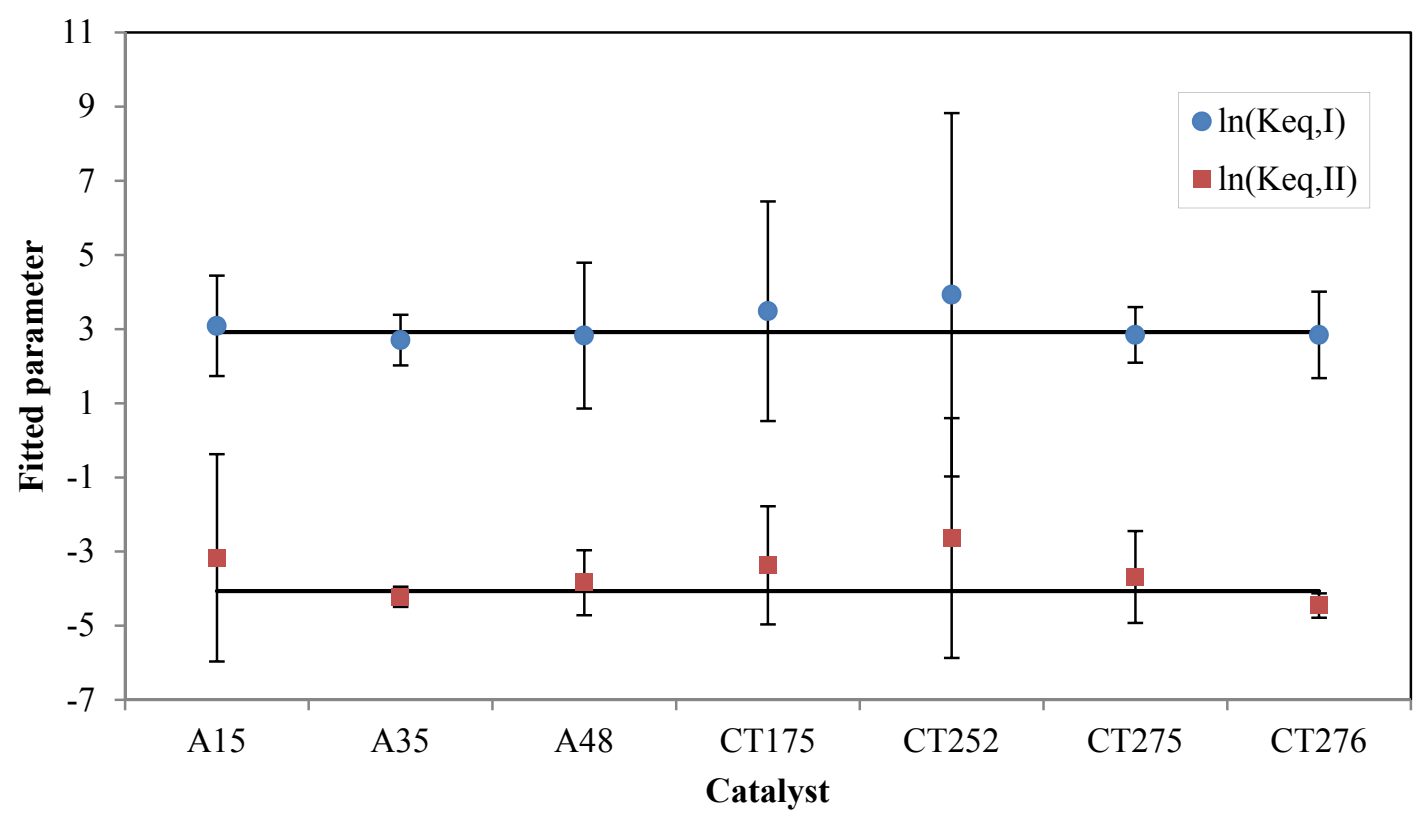

Figure 12: Fitted parameter and weighted mean average values for the equilibrium constants of reactions I and II. Error bars correspond to the standard error of the parameters.

In a second step, all experiments at $373 \mathrm{~K}$ were fitted together to force all resins to fulfill the same values for the equilibrium constants (parameters $k_{2}$ and $k_{4}$ ), but with the rest of the parameters being only dependent of the assayed resin, that is the same value for replicated experiments and different for each resin. In addition, the global fit provides a lower standard error of the estimated parameters, mainly for the equilibrium constants. The obtained results are presented in Table 3.

Table 3. Optimum parameter values, related to kinetic and equilibrium constants, for each resin at $373 \mathrm{~K} . k_{I}$ and $k_{I I}$ are expressed in $\mathrm{L} /(\mathrm{g} \mathrm{min}), \mathrm{k}_{\mathrm{III}}$ in $\mathrm{L}^{2} /(\mathrm{mol} \mathrm{g} \mathrm{min})$, and $K_{\text {eq,I }}$ and $K_{e q, I I}$ are dimensionless .

\begin{tabular}{cccccc}
\hline Catalyst & $\ln \left(\boldsymbol{k}_{\boldsymbol{I}}\right)$ & $\ln \left(\boldsymbol{K}_{\text {eq, }, \boldsymbol{I}}\right)$ & $\ln \left(\boldsymbol{k}_{\mathbf{I I}}\right)$ & $\ln \left(\boldsymbol{K}_{\text {eq,II }}\right)$ & $\ln \left(\boldsymbol{k}_{\mathrm{III}}\right)$ \\
\hline $\mathrm{A} 15$ & $-6.7 \pm 0.2$ & \multirow{2}{*}{$2.9 \pm 0.4$} & $-12.7 \pm 0.2$ & & $-13.66 \pm 0.15$ \\
$\mathrm{~A} 35$ & $-6.88 \pm 0.13$ & & $-12.65 \pm 0.17$ & & $-13.49 \pm 0.10$
\end{tabular}




\begin{tabular}{cccc}
\hline A48 & $-7.2 \pm 0.2$ & $-12.3 \pm 0.3$ & $-13.36 \pm 0.18$ \\
CT175 & $-7.1 \pm 0.3$ & $-12.1 \pm 0.4$ & $-13.62 \pm 0.18$ \\
CT252 & $-7.1 \pm 0.2$ & $-11.8 \pm 0.4$ & $-13.24 \pm 0.17$ \\
CT275 & $-6.49 \pm 0.16$ & $-12.83 \pm 0.16$ & $-12.90 \pm 0.13$ \\
CT276 & $-6.6 \pm 0.2$ & $-12.7 \pm 0.2$ & $-13.21 \pm 0.18$ \\
\hline
\end{tabular}

Finally, in a third step, the temperature dependence of the kinetic and equilibrium constants has been studied. The considered experiments are those carried out at different temperatures for two resins: A35, in the range $353 \mathrm{~K}-393 \mathrm{~K}$, and CT275, in the range $353 \mathrm{~K}-383 \mathrm{~K}$. The considered kinetic model (Equations 5 to 11) has been fitted respectively to the experiments performed at the same temperature and with the same catalyst, what resulted in a set of optimum parameter values for each condition and resin. By considering that kinetic constants follow the Arrhenius law and the thermodynamic dependence of the equilibrium, they can be described as:

$$
\ln (k), \ln \left(K_{e q}\right)=k_{i}=A_{i}+B_{i}\left(1 / T[K]^{-1 / 373.15}\right)
$$

Parameters $A$ and $B$ have been obtained by applying a weighted least squares regression to the optimum parameters, the weighting factor being the reciprocal of the variance of the parameters. The term of temperature $373.15 \mathrm{~K}$ has been included to reduce the correlation between parameters.

As a result, for each resin a temperature dependence of the kinetic constants $k_{I}, k_{I I}$, and $k_{I I I}$ has been found. On the other hand, as equilibrium constants do no depend on the catalyst, they have been fitted irrespectively of the used resin through a weighted linear regression. This plot is shown in Figure 13, where a large error has been observed for $\mathrm{K}_{\mathrm{eq}, \mathrm{I}}$ at higher temperatures. 


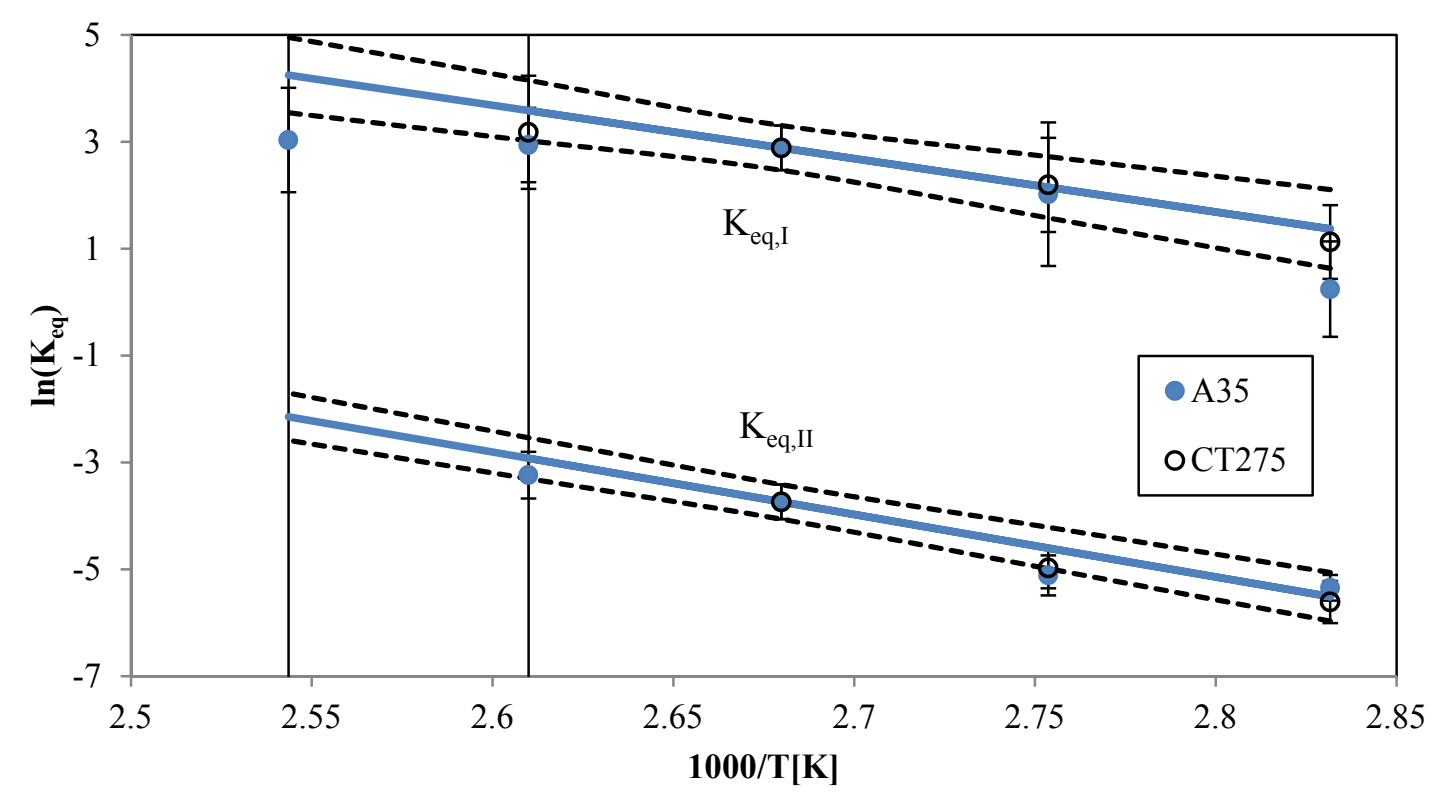

Figure 13. Dependence of the equilibrium constants of reaction I and II over temperature

The results of the temperature dependence of the different constants are as follows:

Catalyst A35:

$$
\begin{aligned}
& \ln \left(k_{I}\left[\frac{\mathrm{L}}{\mathrm{g} \min }\right]\right)=-(6.96 \pm 0.08)-(9.6 \pm 1.1) \cdot 10^{3}\left(\frac{1}{T[K]}-\frac{1}{373.15}\right) \\
& \ln \left(k_{\text {II }}\left[\frac{\mathrm{L}}{\mathrm{g} \min }\right]\right)=-(12.5 \pm 0.2)-(9 \pm 2) \cdot 10^{3}\left(\frac{1}{T[K]}-\frac{1}{373.15}\right) \\
& \ln \left(k_{I I I}\left[\frac{\mathrm{L}^{2}}{\mathrm{~mol} \mathrm{~g} \min }\right]\right)=-(13.2 \pm 0.2)-(10 \pm 3) \cdot 10^{3}\left(\frac{1}{T[K]}-\frac{1}{373.15}\right)
\end{aligned}
$$

Catalyst CT275:

$$
\begin{aligned}
& \ln \left(k_{I}\left[\frac{\mathrm{L}}{\mathrm{g} \min }\right]\right)=-(6.49 \pm 0.03)-(6.7 \pm 0.1) \cdot 10^{3}\left(\frac{1}{T[K]}-\frac{1}{373.15}\right) \\
& \ln \left(k_{I I}\left[\frac{\mathrm{L}}{\mathrm{g} \min }\right]\right)=-(12.69 \pm 0.2)-(10 \pm 4) \cdot 10^{3}\left(\frac{1}{T[K]}-\frac{1}{373.15}\right) \\
& \ln \left(k_{\text {III }}\left[\frac{\mathrm{L}^{2}}{\mathrm{~mol} \mathrm{~g} \min }\right]\right)=-(12.79 \pm 0.10)-(4.5 \pm 1.4) \cdot 10^{3}\left(\frac{1}{T[K]}-\frac{1}{373.15}\right)
\end{aligned}
$$

Equilibrium constants, valid for both resins 


$$
\begin{aligned}
& \ln \left(K_{e q, I}[-]\right)=(2.9 \pm 0.4)-(10 \pm 2) \cdot 10^{3}\left(\frac{1}{T[K]}-\frac{1}{373.15}\right) \\
& \ln \left(K_{e q, I}[-]\right)=-(3.7 \pm 0.3)-(11.7 \pm 0.9) \cdot 10^{3}\left(\frac{1}{T[K]}-\frac{1}{373.15}\right)
\end{aligned}
$$

Despite of the relatively large standard error of the parameters, values in Table 3 and Equations 16 to 23 are considered to be appropriate to describe the evolution of the chemical species of the respective conditions for conversions similar to the obtained in the experiments of the present study. However, to obtain reliable thermodynamic equilibrium properties it is advisable to carry out specific experiments where chemical equilibrium is reached.

\section{Conclusions}

The double bond isomerization and dimerization of 1-octene were feasible over macroreticular acidic ion-exchange resins at $373 \mathrm{~K}$, whereas skeletal isomerization was very scarce. Higher oligomers or cracking products were not detected. Acidity was the most important structural property of the catalysts tested, but not all active sites were accessible for most of the catalysts. The accessible acidity was estimated from 1-octene conversion per active sites, taking as reference the surface sulfonated Amberlyst 46. The selectivity of the reaction can be explained in terms of the accessible acidity, having it a positive effect on $\mathrm{C} 16$ production. Selectivity to dimers and branched isomers increased with increasing temperature but still cracking compounds were not detected. The theoretical equations derived from the proposed kinetic model fit well the experimental results.

\section{Acknowledgements}


Authors thank Rohm \& Haas and Purolite for providing their catalysts. We are also grateful to Repsol YPF for the financial support to this research and for permission to publish this work.

\section{References}

[1] R.J. Quann, L.A. Green, S.A. Tabak, F.J. Krambeck, Chemistry of olefins oligomerization over ZSM-5 catalyst., Industrial and Engineering Chemistry Research 27 (1988) 565-570.

[2] M. Marchionna, M. Di Girolamo, R. Patrini, Light olefins dimerization to high quality gasoline components, Catal Today 65 (2001) 397-403

[3] R. Schmidt, M.B. Welch, B.B. Randolph, Oligomerization of C5 olefins in light catalytic naphtha, Energy and Fuels 22 (2008) 1148-1155.

[4] I. Coleto, R. Roldán, C. Jiménez-Sanchidrián, J.P. Gómez, F.J. Romero-Salguero, Valorization of $\alpha$-olefins: Double bond shift and skeletal isomerization of 1-pentene and 1-hexene on zirconia-based catalysts. Catalysis Today 149 (2010) 275-2무

[5] R. Schwarzer, E. du Toit, W. Nicol, Solid phosphoric acid catalysts: The effect of free acid composition on selectivity and activity for 1-hexene dimerization, Applied Catalysis A: General 369 (2009) 83-89

[6] R. Schwarzer, E. du Toit, W. Nicol, Kinetic model for the dimerization of 1-hexene over a solid phosphoric acid catalyst, Applied Catalysis A: General 340 (2008) 119-124. [7] J. M. Escola, R. Van Grieken, J. Moreno, and R. Rodríguez, Liquid-Phase Oligomerization of 1-Hexene Using Al-MTS Catalysts, Ind. Chem. Eng. Res. 45 (2006) $7409-7414$ 
[8] R. Van Grieken, J.M. Escola, J. Moreno, R. Rodríguez, Nitrogen and sulphur poisoning in alkene oligomerization over mesostructured aluminosilicates (Al-MTS, AlMCM-41) and nanocrystalline n-HZM-5, Applied Catalysis A: General 337 (2008) 173183.

[9] R. Van Grieken, J.M. Escola, J. Moreno, R. Rodríguez, Liquid phase oligomerization of 1-hexene over different mesoporous aluminosilicates (Al-MTS, AlMCM-41 and Al-SBA-15) and micrometer/nanometer HZSM-5 zeolites, Applied Catalysis A: General 305 (2006) 176-188.

[10] A. De Klerk, Oligomerization of 1-hexene and 1-octene over solid acid catalysts, Industrial and Engineering Chemistry Research 44 (2005) 3887-3893

[11] A. De Klerk, Reactivity differences of octenes over solid phosphoric acid, Industrial and Engineering Chemistry Research 45 (2006) 578-584.

[12] N.G. Grigor'eva, S.V. Bubennov, B.I. Kutepov, Oligomerization of $\alpha$-octene catalyzed by zeolites, Catalysis in Industry 3 (2011) 144-150.

[13] P.W.N.M. van Leeuwen, N.D. Clement, M.J.-. Tschan, New processes for the selective production of 1-octene, Coord. Chem. Rev. 255 (2011) 1499-1517 [14] Harmer M.A., Qun Sun. Solid acid catalysis using ion-exchange resins. Applied Catalysis A: General 221 (2001) 45-62

[15] M. Cadenas, R. Bringué, C. Fité, E. Ramírez, F. Cunill, Liquid-phase oligomerization of 1-hexene catalyzed by macroporous ion-exchange resins, Topics in Catalysis 54 (2011) 998-1008

[16] P.F. Siril, H.E. Cross, D.R. Brown. New polystyrene sulfonic acid resin catalysts with enhanced acidic and catalytic properties. Journal of Molecular Catalysis A: Chemical 279 (2008) 63-68 
[17] V.J. Cruz, J.F. Izquierdo, F. Cunill, J. Tejero, M. Iborra, C. Fité, Acid ionexchange resins catalysts for the liquid-phase dimerization/etherification of isoamylenes in methanol or ethanol presence, React Funct Polym 65 (2005) 149-160 